\title{
COVID-19 Disease Outcomes: Does Gastrointestinal Burden Play a Role?
}

\author{
Mona A Hegazy' \\ Rania Mohamed Lithy ${ }^{2}$ \\ Hoda M Abdel-Hamid ${ }^{3}$ \\ Mahmoud Wahba' \\ Omar Ahmed Ashoush' \\ Mohamed Tharwat Hegazy (D) \\ Maha Hossam El- \\ Din Ibrahim (D) \\ Dalia Abdelfatah (iD) ${ }^{4}$ \\ Ahmed Abdelghani ${ }^{1}$ \\ 'Internal Medicine Department, Faculty \\ of Medicine, Cairo University, Cairo, \\ Egypt; ${ }^{2}$ Endemic Medicine Department, \\ Faculty of Medicine, Cairo University, \\ Cairo, Egypt; ${ }^{3}$ Chest Diseases \\ Department, Faculty of Medicine, Cairo \\ University, Cairo, Egypt; ${ }^{4}$ Biostatistics and \\ Cancer Epidemiology Department, \\ National Cancer Institute, Cairo \\ University, Cairo, Egypt
}

\begin{abstract}
Background: The novel coronavirus disease 2019 presents an urgent threat to global health. As the epidemic grows, prognosis prediction is essential for monitoring risky patient. It is thus important to consider gastrointestinal manifestations and the duration of symptoms as predictors of prognosis. Our aim was to determine the correlation of gastrointestinal symptoms and laboratory markers with disease outcomes and whether symptom duration varies substantially between patients. We also undertook this study to determine the optimal time to predict COVID-19 outcome.
\end{abstract}

Patients and Methods: A total of 190 patients with polymerase chain reaction-confirmed COVID-19 were followed up until recovery. We proposed a correlation between gastrointestinal symptoms and disease severity (based on clinical data, and diagnostic investigations) to estimate the duration of symptoms as a predictor of COVID-19 prognosis.

Results: The prevalence of gastrointestinal symptoms was $49.5 \%$, consisting mainly of diarrhea in $27.9 \%$ of patients. In addition, a longer disease duration and higher temperature were observed in patients with diarrhea. Symptom duration was variable, with a median of 12 days and a range of 1-55 days. Statistical analysis indicated that patients with a duration of symptoms $\geq 12$ day had more severe symptoms and a worse prognosis. Patients who complained of diarrhea had 2.7 times the odds of a longer duration of symptoms, and those with a history of chronic lung disease have 7.2 times the odds of a longer duration of symptoms.

Conclusion: GIT manifestations (mainly diarrhea) and the duration of symptoms of COVID-19 provide prognostic evidence of COVID-19 outcomes, irrespective of earlier categorization by the World Health Organization. Thus, patients with mild symptoms who present with diarrhea and a duration of symptoms longer than 12 days are expected to have a worse prognosis.

Keywords: COVID-19, GIT manifestations, symptoms duration, prognosis

\section{Plain Language Summary}

In our study, we propose a prognostic classification based on the duration of illness and presence of gastrointestinal manifestations (mainly diarrhea) that can be applied to estimate COVID-19 disease outcome. A median symptom duration of 12 days was found to predict a worse patient prognosis.

\section{Introduction}

Correspondence: Mona A Hegazy Internal Medicine Department, Faculty of Medicine, Cairo University, P.O. Box Number: 12566, 6th October City, Giza, Egypt

Tel +20l00l42I55I

Email monahegazy@cu.edu.eg
The novel coronavirus disease (COVID-19) presents an urgent threat to global health, and has put a strain on healthcare systems worldwide. ${ }^{1}$ The large number of infected persons has resulted in unmet medical demands, and many countries are facing a health crisis. ${ }^{2}$ 
The early identification and prompt treatment of critical COVID-19 cases are crucial. ${ }^{3}$ Previous studies have suggested that older age, and higher SOFA score on admission, and elevated D-dimer levels are associated with increased mortality. ${ }^{4}$

Although COVID-19 patients typically present with respiratory symptoms, some patients had gastrointestinal (GI) manifestations, including diarrhea, vomiting, and colicky abdominal pain, during the disease course. ${ }^{5} \mathrm{~A}$ study by Zhou et al suggests that GI symptoms are common clinical symptoms in patients with novel coronavirus-infected pneumonia. ${ }^{6}$ In addition, it may be the first symptom and patients show no response to treatment with common therapeutic agents. ${ }^{7}$

Previous clinical studies showed conflicting finding regarding the presence of gastrointestinal symptoms and poor outcomes. Patients with gastrointestinal symptoms had a significantly longer time from onset to admission than those without gastrointestinal symptoms as they did not initially present with typical respiratory symptoms and so they did not receive timely diagnoses and treatment. ${ }^{8}$

The duration of symptoms has not been well characterized and a few studies have suggested that the clinical presentation and patient characteristics could predict the duration of symptoms and viral shedding in COVID-19 infection. ${ }^{9,10}$

Thus, we conducted the present study to determine the correlation of GI symptoms and laboratory markers with disease outcomes. Moreover, because the duration of symptoms varies substantially between patients, we aimed to determine the optimal time to predict COVID19 outcome.

\section{Patients and Methods}

\section{Study Design}

This retrospective observational study was conducted on COVID-19 patients (positive polymerase chain reaction [PCR] results via nasopharyngeal swab) between June 1 and mid July 2020. Patients were recruited from Kasr AlAini School of Medicine, Cairo University Hospitals.

We included all adult patients $(18-75$ years $)$ suffering from mild or moderate COVID-19 infection according to World Health Organization (WHO) criteria for the clinical management of COVID-19. ${ }^{11}$ Patients were excluded if they had severe COVID-19 disease, were hospitalized, or had unavailable nasopharyngeal swab results.
According to WHO classification, COVID-19 infection was categorized into mild, moderate or severe infection. Mild COVID-19 defines as respiratory symptoms without evidence of pneumonia or hypoxia, while moderate or severe infection defined as presence of clinical and radiological evidence of pneumonia. In moderate cases, $\mathrm{SpO} 2$ $\geq 90 \%$ on room air while one of the following was required to define the severe cases: respiratory rate $>30$ breaths $/ \mathrm{min}$ or $\mathrm{SpO} 2<90 \%$ on room air. ${ }^{11}$

Treatment protocol followed the recommendations of the Egyptian Ministry of Health and Population Management, which was approved by the scientific COVID-19 committee of Faculty of Medicine, Cairo University. $^{12}$

\section{Data Collection}

We followed up 190 COVID-19 patients until recovery of symptoms to determine the correlation between GI symptoms and disease severity and to estimate the duration of symptoms as a predictor of COVID-19 prognosis. We retrospectively correlated the duration of symptoms with prognosis and detected the best time to predict the patients' worse prognosis. We collected clinical data including patient demographics (age, gender, body mass index [BMI], underlying comorbidities, clinical symptoms, and vital signs).

Clinical symptoms were recorded using a yes/no checklist that asked every patient about constitutional symptoms (fever, fatigue), respiratory symptoms (cough, dyspnea), neurological symptoms (headache, loss of taste, and loss of smell), and GI symptoms (nausea, vomiting, abdominal pain, and diarrhea). Diarrhea was defined in this study according to the following WHO criteria: three or more loose stools/day or an increase in the number of motions as compared with usual.

Patients were followed up throughout their illness via phone calls and scheduled commercial video chat platforms until they achieved full recovery of their symptoms, and some patients until they tested negative by PCR (according to their job's return policy).

Laboratory assessment consisted of complete blood examination, kidney and liver biochemistry (aspartate aminotransferase, alanine aminotransferase, and total bilirubin), fasting blood glucose, serum C-reactive protein (CRP), D-dimer, ferritin, and lactate dehydrogenase, which were collected within 24 hours of symptom onset. Initial chest $\mathrm{CT}$ was performed and result recorded. 


\section{Statistical Analysis}

Data management and analysis were performed using the Statistical Package for Social Sciences version 25. Numerical data were summarized using means and standard deviations or medians and ranges, as appropriate. Categorical data were summarized as numbers and percentages. Numerical data were explored for normality using the Kolmogorov-Smirnov test and Shapiro-Wilk test.

Chi-square or Fisher tests were used for betweengroups comparisons of categorical data, as appropriate. Comparisons between two groups for normally distributed numeric variables were performed using the Student's $t$-test, while for nonnormally distributed numeric variables, comparisons were done by Mann_Whitney test. Logistic regression was performed to provide the adjusted odds ratio (OR) and the magnitude of the effect of different risk factors. The OR and $95 \%$ confidence interval $(95 \%$ CI) were also calculated (a 95\% CI that does not contain 1.0 is considered significant). All tests were two sided. $P$ values $<0.05$ were considered significant. ${ }^{13}$

\section{Ethical Considerations}

Informed consent was obtained from all patients before study inclusion. The study design followed the requirements of Revised Helsinki Declaration of biomedical ethics $^{14}$ and was approved by the Research Ethics Committee, Faculty of Medicine, Cairo University (REC n-86-2020; August 16, 2020). The trial is registered on clinical trial.gov of the US National Library of Medicine (cinicalTrial.gov identifier: NCT04554979).

\section{Results}

A total of 190 COVID-19 patients were included in the present study; 113 patients were considered mild cases and 77 moderate cases. The mean patient age was significantly different between both mild and moderate patients (37 and 45 years of age, respectively); $5.3 \%$ of mild cases were younger than 50 years, and $37.6 \%$ of moderate cases were aged 50 years or older $(p=0.001)$. Ninety-four of the 190 COVID-19 patients were men; $46.8 \%$ of them presented by moderate disease severity, whereas only $30.8 \%$ of the females were moderate cases $(p=0.033)$.

Moderate cases showed significantly higher metabolic comorbidities, including higher BMI, frequency of diabetes, and hypertension $(p=0.016,0.003$, and 0.027 , respectively). Ten moderate SARS-COV-2-infected patients $(13 \%)$ had chronic lung disease, whereas only
$4.4 \%$ of the mild cases had the same medical condition $(p=0.022)$.

Of the COVID-19 patients, $49.5 \%$ had nonspecific GIT manifestations of "anorexia and nausea" in addition to respiratory and constitutional symptoms. Diarrhea was the main presentation in $53(27.9 \%)$ patients and was associated with vague paraumbilical pain in 11 patients, and only two patients presented with vomiting. Diarrhea was distributed almost equally between mild and moderate cases according to the WHO classification $(27.4 \%$ and $28.6 \%$, respectively). A higher core temperature was recorded in patients who presented with diarrhea irrespective of the severity of SARS-COV-2 infection severity ( $p=$ 0.016; Figure 1).

Table 1 shows the laboratory investigations for both mild and moderate cases. Only 81 patients were retested by PCR to confirm the cure according to their job's returnto-work policy (54 mild and 27 moderate cases). The median duration to a negative PCR test was $26(8-58)$ in mild cases, and $27(10-45)$ in moderate ones. Although the duration of symptoms was variable, with a median of 12 days (range, 1-55 days), the median was statistically lower in mild cases at 10 days (range, $1-55$ days), whereas it was 15 days $(4-55$ days) in moderate cases $(p<0.001)$.

From a statistical point of view, using a median duration of symptoms of 12 days in SARS- COV-2-infected patients, the patients were further subdivided into two groups bases symptom duration: group I $(\mathrm{n}=94)$ included patients whose symptoms lasted $<12$ days $(73.4 \%$ mild and $26.6 \%$ moderate cases), and group II $(\mathrm{n}=96)$ consisted of patients with symptoms lasting $\geq 12$ days symptom $(50 \%$ moderate cases; $p=0.001)$. The relation between duration of symptom recovery and confirmed cured via PCR test was significant irrespective of the disease severity classification (Table 2 and Figure 2). This finding confirms that the patients' prognosis can be determined using the 12-days symptom duration and its correlation with COVID-19 disease severity.

Stratification of the demographic data of the two groups based on the duration of symptoms showed the following: The mean patient age was 38 years in group I and 43 years in group II $(p=0.007)$ pass hand on hand with the age in mild and moderate patients, respectively. Of the cases, $84 \%$ of cases presented within 12 days or less duration and were less than 50 years of age in group I; this proportion decreased to $71.9 \%$ with a $\geq 12$ days duration of symptoms $(p=0.033)$. However, there was no significant difference between the two groups in gender 


\section{GIT manifestations (No) GIT manifestations (Yes)}

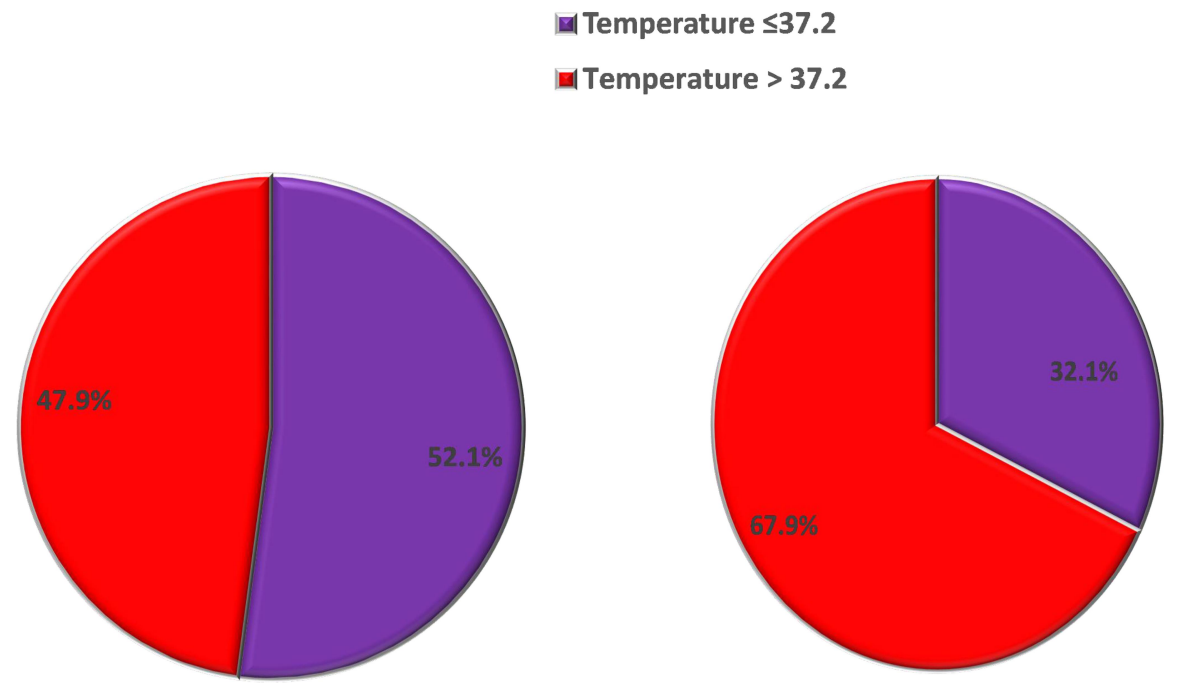

Figure I Core temperature in relation to GIT manifestation.

or comorbidities, except for chronic lung disease, which remains the cornerstone: 2 of 94 (1.9\%) patients who had a duration of symptoms $<12$ days had a chronic lung diseases, whereas $13.5 \%$ of the patients who presented with a symptom duration of $\geq 12$ days had a chronic lung disease $(p=0.004)$.

Diarrhea was reported in 36 (37.5\%) COVID-19 patients with symptom lasting $\geq 12$ days, whereas only 17 (18.1\%) of patients with symptoms of $<12$ days had diarrhea $(p=0.003)$, irrespective of the WHO's COVID-19 disease severity classification, whereby our patients had a matched incidence of diarrhea in mild and moderate cases, as mentioned previously (Figure 3). It is worth noting that $13.2 \%$ of our patients with diarrhea had diabetes.

A higher temperature was recorded in patients who presented with diarrhea irrespective of their SARS-COV2 infection severity: $34 \%$ of patients with a high core temperature $\left(>37.2^{\circ} \mathrm{C}\right)$ had diarrhea, whereas only $18.3 \%$ of patients with normal temperature complained of diarrhea $(p=0.029)$.

Lymphocytic count, CRP, and ferritin were significantly correlated not only with disease severity but also with the duration of symptoms, as demonstrated in (Table 3 ). The median CRP level was higher in patients with diarrhea (9.3 versus $5 \mathrm{mg} / \mathrm{L}$ ), and median lymphocytic count was lower in patients with diarrhea (2070 versus $2100 / \mathrm{uL}$ ).

To measure the independent effect of all factors that affect SARS-COV-2 infection severity, we conducted a multivariate logistic regression for the prediction of COVID-19 disease severity. Factors that had a significance level less than 0.100 were selected for inclusion in the stepwise logistic regression. Our results showed that diarrhea and chronic lung disease were the independent factors associated with the outcome of SARS-COV-2 infection (Table 4). Hence, patients who complained of diarrhea had 2.7 times the odds of a longer duration of symptoms. Moreover, patients with a history of chronic lung disease had 7.2 times the odds of a longer duration of symptoms.

\section{Discussion}

The WHO prospectively classifies disease severity based on clinical symptoms and signs of pneumonia together with chest imaging. However, GIT symptoms and duration of illness were not included in that classification, although GIT symptoms were the principle presentation in many patients and the duration of symptoms varies substantially between patients irrespective of their disease severity. These factors raise the question as to whether we can classify disease severity by the presence of GIT manifestations together with respiratory symptoms from the start, as well as whether a prognostic classification based on symptom duration could add another helpful disease categorization.

In this study, we included 113 mild and 77 moderate patients, based on the WHO classification. Patients with moderate COVID-19 were significantly older than mild cases $(P=$ 0.001 ), indicating that age can be a predictor of disease 
Table I Laboratory Characteristics of the Studied Patients

\begin{tabular}{|c|c|c|c|c|}
\hline & All $(n=190)$ & Mild $(n=113)$ & Moderate $(n=77)$ & \multirow[t]{2}{*}{$P$ value } \\
\hline & \multicolumn{3}{|c|}{ Mean \pm SD } & \\
\hline Hemoglobin (g/dl) & $13.3 \pm 1.8$ & $13.2 \pm 1.8$ & $13.4 \pm 1.8$ & 0.446 \\
\hline Platelets $\left(10^{3} / \mathrm{cmm}\right)$ & $232.8 \pm 78.6$ & $230.0 \pm 72.0$ & $237.3 \pm 88.3$ & 0.522 \\
\hline Total leukocyte count $(/ \mathrm{cmm})$ & $6591.8 \pm 2750.4$ & $6735.8 \pm 2680.6$ & $6366.4 \pm 2859.0$ & 0.355 \\
\hline Neutrophil \% & $53.5 \pm 14.3$ & $51.6 \pm 13.5$ & $56.4 \pm 15.0$ & 0.018 \\
\hline Urea (mg/dl) & $27.2 \pm 10.3$ & $26.5 \pm 7.8$ & $28.4 \pm 13.7$ & 0.264 \\
\hline Creatinine (mg/dl) & $0.9 \pm 0.2$ & $0.8 \pm 0.2$ & $0.9 \pm 0.2$ & 0.025 \\
\hline \multirow[t]{2}{*}{ LDH (U/L) } & $245.4 \pm 107.0$ & $245.7 \pm 115.7$ & $244.9 \pm 92.9$ & 0.960 \\
\hline & \multicolumn{3}{|c|}{ Median (range) } & \\
\hline Lymphocytic count $(/ \mu \mathrm{L})$ & $2075(504-5900)$ & $2240(820-5900)$ & $1784(504-4950)$ & 0.002 \\
\hline Lymphocytes \% & $35(5-68)$ & $35.2(9.0-68.0)$ & $35.0(5.0-63.0)$ & 0.134 \\
\hline$N / L$ ratio & $1.5(0.1-17.8)$ & $1.5(0.1-9.7)$ & I.8 (0.3-I7.8) & 0.019 \\
\hline $\begin{array}{l}\text { Absolute neutrophilic count } \\
(/ \mu \mathrm{L})\end{array}$ & $3200(400-15,460)$ & $3368.5(400-9900)$ & $3056(600-15,460)$ & 0.851 \\
\hline AST (U/L) & $23(8-69)$ & $20.5(8-69)$ & $27(11-67)$ & $<0.001$ \\
\hline ALT (U/L) & $22(5-139)$ & $22(5-89)$ & $24.5(10-139)$ & 0.012 \\
\hline Total bilirubin $(\mathrm{mg} / \mathrm{dl})$ & $0.4(0.2-1.7)$ & $0.4(0.2-1.2)$ & $0.5(0.2-1.7)$ & 0.011 \\
\hline Direct bilirubin (mg/dl) & $0.1(0-0.7)$ & $0.1(0-0.7)$ & $0.1(0-0.6)$ & 0.086 \\
\hline CRP $(\mathrm{mg} / \mathrm{L})$ & $6(0.1-2 \mid 6)$ & $4(0.1-216)$ & $12(0.2-166)$ & $<0.001$ \\
\hline Ferritin (ng/mL) & II 8.5 (4-1084) & $100(4-657)$ & $160(9-1084)$ & $<0.001$ \\
\hline D-Dimer (mg/L) & $0.4(0.1-9.0)$ & $0.3(0.1-2.6)$ & $0.4(0.1-9.0)$ & 0.539 \\
\hline
\end{tabular}

Notes: $P<0.05$ is considered significant.

Abbreviations: ALT, alanine aminotransferase; AST, aspartate aminotransferase; CRP, C-reactive protein; N/L, neutrophil-to-lymphocyte ratio; SD, standard deviation.

Table 2 Cure (Negative PCR) in Relation to Duration of Symptoms

\begin{tabular}{|l|l|l|l|l|}
\hline \multicolumn{2}{|l|}{} & Group I (<I2 Days) $\mathbf{n}=\mathbf{4 4}$ & Group II ( $\geq \mathbf{I} \mathbf{2}$ Days) $\mathbf{n}=\mathbf{3 7}$ & $\mathbf{P}$ value \\
\hline CURE (-VE PCR), $\mathbf{n}=8$ I DATE & Mean \pm SD & $22 \pm 10$ & $29 \pm 9$ & 0.002 \\
& Median (range) & $19(8-48)$ & $28(I 0-458)$ & \\
\hline
\end{tabular}

severity, which was in agreement with an earlier published study. ${ }^{15}$ Of the men, $46.8 \%$ tended to have moderate infection, whereas $30.8 \%$ of women had the same degree of disease severity $(P=0.033)$. This could be explained by the biological differences in their immune systems mediated by many factors, including sex hormones and the high expression of angiotensin-converting enzyme receptors. ${ }^{16}$

Moderate cases showed a significantly higher frequency of metabolic comorbidities (obesity, diabetes, and hypertension), and this finding was in agreement with previous studies. ${ }^{17,18}$ ACE2 is expressed in lung alveolar type 2 cell and can also be found in the upper esophagus and in enterocytes in the ileum and colon. ${ }^{19}$ The present study revealed that $13 \%$ of moderate and $4 \%$ of mild patients had chronic lung disease $(P=0.022)$, and those patients with a history of chronic lung disease had a longer duration of symptoms ( $\geq 12$ days), mostly because of poor underlying lung reserve, and increased expression of ACE2 receptor in small airways. 


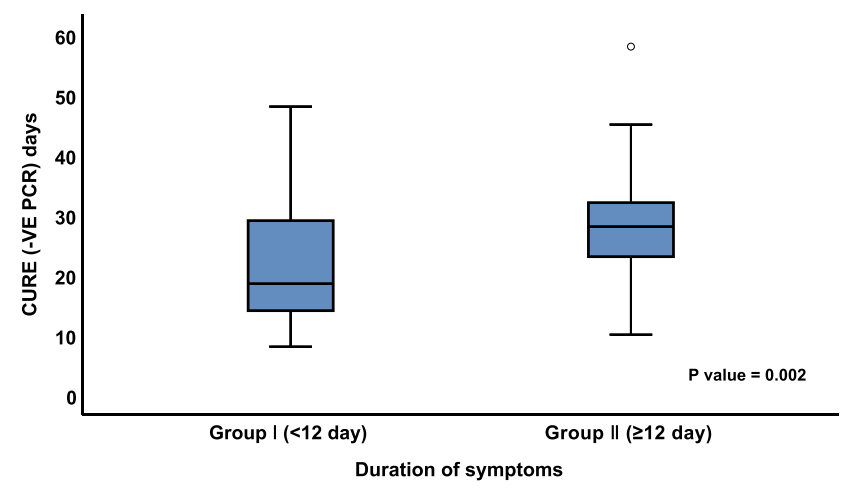

Figure 2 Cure (negative PCR) in relation to the duration of symptoms.

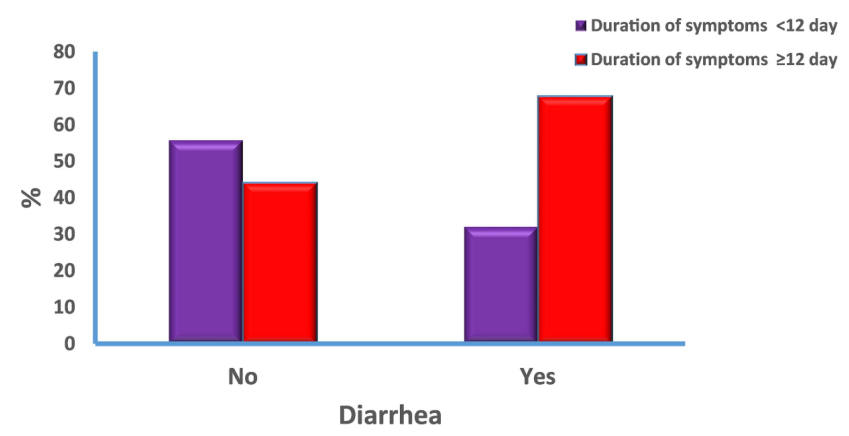

Figure 3 Diarrhea in relation to duration of symptoms.

Clinicians should be aware that COVID-19 can present with principally GI symptoms and not solely constitutional and respiratory symptoms. In the present study, we found that the prevalence of GIT symptoms (anorexia and nausea) was 49.5\% in COVID-19 patients in addition to respiratory and constitutional symptoms. Diarrhea was the main presentation in $53(27.9 \%)$ patients, which was associated with vague paraumbilical pain in 11 patients $(5.8 \%)$ and vomiting in 2 patients (1\%). Diarrhea was distributed almost equally between mild and moderate cases according to the WHO classification (27.4\% and $28.6 \%$, respectively), occurring in $37.5 \%$ of patients with a symptom duration $\geq 12$ days. Between $50.5 \%$ and $61.3 \%$ of COVID-19 patients in China and the United States were reported to have GIT symptoms. ${ }^{5,8}$ In Wuhan, China, $31 \%$ of patients with SARS-CoV-2 pneumonia had diarrhea, and they had a greater viral RNA load in the stool (69\% versus 17\%); elimination of SARS-CoV-2 from the stool takes longer than elimination from the nose and throat. ${ }^{20}$

A meta-analysis performed by D'Amico et al revealed a great variability among published studies in the percentage of patients with diarrhea, ranging from $2 \%$ to $50 \%$ of cases. A pooled analysis of the available data showed an overall diarrhea rate of about $10 \%$ in COVID-19 patients. $^{21}$
The great variability between studies could be explained by the involvement of nonspecific symptoms, the subjective nature of some symptoms, and the lack of an accurate definition of diarrhea. In the current study, diarrhea was defined based on the WHO criteria of three or more loose/liquid stools per day or an increase in the number of motions compared with the usual. ${ }^{22}$ In a study by Jin et al, the authors reported sequence mutation in S2 (the portion of the S protein that promotes binding of the virus to host cells), which could explain the increased GI symptoms in the later phase of this virus outbreak and their novel epidemiological and clinical features. ${ }^{23}$

The pathophysiology of the enteric affection in COVID-19 is not completely known. SARS-COV-2 can enter the digestive system through ACE2-expressing cells, and the cytokine storm associated with severe COVID-19 infection can cause hypoxia-induced bowel ischemia and promote diarrhea. $^{24}$

In our study, we found that the duration of illness was variable, with a median of 12 days. Despite mild infection, a longer duration to recovery ( $\geq 12$ days) was noted in $50.5 \%$ of mild patients, whereas a shorter duration of recovery ( $<12$ days) was observed in $26.6 \%$ of moderate patients. We proposed that classifying patients based on duration of illness could predict disease outcome. Our study revealed that patients younger than 50 years tend to have a shorter duration of symptoms $(p=0.033)$. We also found that there was no significant difference between the two groups in gender or comorbidities, and this result was in accordance with the findings of O'Keefe et al. ${ }^{25,26}$

Interesting enough, the present study found that a longer duration of disease and higher recorded core temperature were observed in patients who presented with diarrhea: $37.5 \%$ of patients who presented with $\geq 12$ days of symptoms had diarrhea as compared with only $18.1 \%$ of those with $<12$ days of symptoms. Thus, $67.9 \%$ of patients who presented with diarrhea had symptoms that lasted for $\geq 12$ days ( $p=0.003$ ). Notably, diarrhea was present in $34 \%$ of patients with a recorded elevated core temperature $>37.2^{\circ} \mathrm{C}$, versus only $18.3 \%$ of patients who presented with a normal temperature $(p=0.029)$. An earlier study published by Jin et al reported a significantly higher rate of $\left(>38.5^{\circ} \mathrm{C}\right)$ fever in patients with COVID-19 with GI symptoms as compared with those without GI symptoms. ${ }^{23}$

The presence of GI symptoms and poor outcomes (disease severity and duration to recovery) was studied earlier in Wuhan, China, with a notable difference found in the duration of illness. Of the patients with COVID-19, 29.3\% had diarrhea, and those patients with GI symptoms 
Table 3 Laboratory Characteristics of the Studied Groups

\begin{tabular}{|c|c|c|c|}
\hline & Group I (<I2 Days) Mean \pm SD & Group II ( $\geq$ I 2 Days) Mean \pm SD & $P$ value \\
\hline Hemoglobin $(g / d l)$ & $13.3 \pm 1.9$ & $13.2 \pm 1.7$ & 0.493 \\
\hline Total leukocyte count (/cmm) & $6791.5 \pm 2666.8$ & $6345.9 \pm 2795.8$ & $0.26 I$ \\
\hline Platelets $\left(10^{3} / \mathrm{cmm}\right)$ & $230.7 \pm 72.5$ & $236.2 \pm 86.9$ & 0.638 \\
\hline Neutrophil \% & $53.2 \pm 12.7$ & $53.8 \pm 16.0$ & 0.792 \\
\hline Urea (mg/dl) & $27.4 \pm 7.8$ & $27.6 \pm 12.5$ & 0.923 \\
\hline Creatinine (mg/dl) & $0.9 \pm 0.2$ & $0.9 \pm 0.2$ & 0.765 \\
\hline \multirow[t]{2}{*}{ LDH $(\mathrm{U} / \mathrm{L})$} & $255.3 \pm 98.4$ & $239.7 \pm 117.5$ & 0.354 \\
\hline & \multicolumn{2}{|c|}{ Median (range) } & \\
\hline Lymphocyte count $(/ \mu \mathrm{L})$ & $2300(820-5900)$ & $1890(504-5090)$ & 0.008 \\
\hline Lymphocytes \% & $35.9(9.0-63.0)$ & $34.0(5.0-68.0)$ & 0.330 \\
\hline Absolute Neutrophil count $(/ \mu \mathrm{L})$ & $3268.5(700-9825)$ & $3108(400-15,460)$ & 0.498 \\
\hline Neutrophil/lymphocyte ratio & I.5 (0.4-9.7) & I.8 (0.1-17.8) & 0.221 \\
\hline Monocytes \% & $8.7(0.5-18.6)$ & $8.1(0.4-17.2)$ & 0.960 \\
\hline AST (U/L) & $23(8-55)$ & $23(10-69)$ & 0.657 \\
\hline ALT (U/L) & $22(5-89)$ & $23(7-139)$ & 0.803 \\
\hline Total bilirubin (mg/dl) & $0.4(0.2-1.2)$ & $0.5(0.2-1.7)$ & 0.272 \\
\hline Direct bilirubin (mg/dl) & $0.1(0-0.7)$ & $0.1(0-0.6)$ & 0.692 \\
\hline CRP (mg/L) & $4(0.1-216)$ & $9.6(0.2-166)$ & 0.002 \\
\hline Ferritin $(\mathrm{ng} / \mathrm{mL})$ & $109.7(4-402)$ & $142(6-1084)$ & 0.043 \\
\hline D-dimer (mg/L) & $0.3(0.1-2.6)$ & $0.4(0.1-4.0)$ & 0.139 \\
\hline
\end{tabular}

Notes: $P<0.05$ is considered significant.

Abbreviations: ALT, alanine transaminase; AST, aspartate transaminase; CRP, C- reactive protein; LDH, lactate dehydrogenase.

Table 4 Multivariate Logistic Regression Analysis for the Significant Variables

\begin{tabular}{|l|l|l|l|l|l|}
\hline & B & S.E. & OR & 95\% CI for OR & P value \\
\hline Diarrhea & 1.0 & 0.3 & 2.7 & $1.4-5.3$ & 0.004 \\
Chronic lung disease & 2.0 & 0.8 & 7.2 & $1.6-33.4$ & 0.012 \\
Constant & -0.4 & 0.2 & 0.7 & NA & 0.045 \\
\hline
\end{tabular}

Notes: $P \leq 0.05$ is considered significant.

Abbreviations: $\mathrm{B}$, regression coefficient; $\mathrm{Cl}$, confidence interval; OR, odds ratio; SE, standard error.

also had a longer hospital stay (9 versus 7.3 days) and more severe form of COVID-19 infection. ${ }^{8}$ Moreover, in another study, patients who presented with diarrhea (3 of 33 SARS-COV-2 patients) required $>10$ days of recovery.${ }^{26}$ However, Wang et al reported that diarrhea was found in only $10.1 \%$ of their cases, and they reported no association between diarrhea and patient outcome. ${ }^{27}$
We found a higher recorded median CRP (9.3 versus 5 $\mathrm{mg} / \mathrm{L})$ and lower lymphocytic count $(2070 / \mu \mathrm{L}$ versus $2100 / \mu \mathrm{L}$ ) in patients with diarrhea, but the difference was not significant. In a multicenter study of 191 patients by Zhou et al, the presence of GI symptoms was associated with elevated CRP, elevated alanine aminotransferase and lower hemoglobin levels when compared with 
patients without GI symptoms ${ }^{4,28}$ Thus, until now, the data regarding the presence of GIT manifestations and their direct relationship to laboratory parameters has not been clear and requires additional verification.

In our study, the mean to a negative PCR test was 26 days (range, 8_58 days), Campioli et al reported that the median time from symptom onset to the first negative PCR test was 23 days (interquartile range $=12$ days), which suggests that testing after 3 weeks of symptoms could be beneficial for the detection of cessation of viral RNA shedding. ${ }^{28}$ We also observed that the duration of symptom recovery was not associated with the confirmed cure by PCR test, and this was of statistical significance regardless of disease severity $(P=0.002)$.

Multivariate logistic regression for the prediction of COVID-19 disease severity revealed that diarrhea and chronic lung disease were the independent factors associated with SARS-COV-2 infection outcome. Patients with a history of chronic lung disease had a 7.2 times greater risk of a longer duration of symptoms, and the GIT burden resulted in patients presenting with diarrhea having a 2.7 times greater risk of a longer duration of symptoms and thus had a strong impact on COVID-19 outcomes.

We must mention some limitations of the present study. The included sample was relatively small sample, with unequal distribution according to disease severity classification by the WHO. This was also a single-center study, and we could not correlate the presence of COVID-19 RNA in the stool with GI symptoms, since this test is not routinely performed in our institution.

We found that the high prevalence of GI symptoms (mainly diarrhea) in SARS-COV-2-infected patients demonstrated the GIT burden in COVID-19 disease. In addition, we propose a prognostic classification based on the duration of illness that can be applied to estimate disease outcome, with 12 days as the median duration for predicting patients' worse prognosis. Much concern should be directed toward clarifying the GIT burden of patients with COVID-19 disease via large-scale studies and its relationship with seasonal and climate variations across different countries. Moreover, diarrhea should be considered in a new COVID-19 categorization of severity in the near future.

\section{Conclusion}

We reported the prevalence of gastrointestinal symptoms in SARS-COV2 infected patients demonstrating a gastrointestinal burden in COVID-19 disease. GI manifestations (mainly diarrhea) and our proposed new a predictor of severity based on duration of illness " 12 days" that can be applied to estimate disease outcome, added to the prognostic evidence of disease outcomes irrespective of the earlier mild, moderate and severe categorization by WHO. When presenting with diarrhea and a longer duration of symptoms ( $\geq 12$ days), even patients with mild cases have a worse prognosis. And more efforts should be made to clarify the full spectrum of GI burden in COVID-19.

\section{Acknowledgments}

The authors would like to thank the frontline medical staff of Kasr Alainy medical school for their bravery and efforts in SARS-COV-2 prevention and control.

\section{Funding}

All authors declare the absence of any financial or personal relationships with other people or organizations that could inappropriately influence and bias the work.

\section{Disclosure}

The authors report no conflicts of interest in this work.

\section{References}

1. Sohrabi C, Alsafi Z, O'Neill N, et al. World Health Organization declares global emergency: a review of the 2019 novel coronavirus (COVID-19). Int $J$ Surg. 2020;76:71-76. doi:10.1016/j. ijsu.2020.02.034

2. Ji D, Zhang D, Xu J, et al. Prediction for progression risk in patients with COVID-19 pneumonia: the CALL score. Clin Infect Dis. 2020;71 (6):1393-1399. doi:10.1093/cid/ciaa414

3. Chen N, Zhou M, Dong X, et al. Epidemiological and clinical characteristics of 99 cases of 2019 novel coronavirus pneumonia in Wuhan, China: a descriptive study. Lancet. 2020;395(10223):507513. doi:10.1016/S0140-6736(20)30211-7

4. Zhou F, Yu T, Du R, et al. Clinical course and risk factors for mortality of adult inpatients with COVID-19 in Wuhan, China: a retrospective cohort study. Lancet. 2020;395(10229):1054-1062.

5. Han C, Duan C, Zhang S, et al. Digestive symptoms in COVID-19 patients with mild disease severity; clinical presentation, stool viral RNA testing, and outcomes. Am J Gastroenterol. 2020;115(6):916923.

6. Zhou Z, Zhao N, Shu Y, Han S, Chen B, Shu X. Effect of gastrointestinal symptoms in patients with COVID-19. Gastroenterology. 2020;158(8):2294-2297. doi:10.1053/j.gastro.2020.03.020

7. Hormati A, Shahhamzeh A, Afifian M, Khodadust F, Ahmadpour S. Can COVID-19 present unusual GI symptoms? J Microbiol Immunol Infect. 2020;53(3):384-385. doi:10.1016/j.jmii.2020.03.020

8. Pan L, Mu M, Yang P, et al. Clinical characteristics of COVID-19 patients with digestive symptoms in Hubei, China: a descriptive, crosssectional, multicenter study. Am J Gastroenterol. 2020;115(5):766773. doi:10.14309/ajg.0000000000000620

9. Tian S, Chang Z, Wang Y, et al. Clinical characteristics and reasons for differences in duration from symptom onset to release from quarantine among patients with COVID-19 in Liaocheng, China. Front Med (Lausanne). 2020;7:210. doi:10.3389/fmed.2020.00210 
10. Lu Y, Li Y, Deng W, et al. Symptomatic infection is associated with prolonged duration of viral shedding in mild Coronavirus disease 2019: a retrospective study of 110 children in Wuhan. Pediatr Infect Dis J. 2020;39(7):e95-e99. doi:10.1097/INF.0000000000002729

11. World Health Organization. Clinical Management of COVID-19: Interim Guidance. World Health Organization; 2020:13-15.

12. Masoud HH, Elassal G, Zaky S, et al. Management Protocol for COVID-19 Patients Version 1.4/30th May 2020 Ministry of health and population (MOHP), Egypt. In: Coronavirus Disease 2019 (COVID-19), SARS COV2 Management Guide line. Cairo: Egypt Ministry of Health and Population; 2020. Available from: https:// www.researchgate.net/publication/344078546 Management Protocol_for_COVID-19_Patients_Version_1430th_May_2020_ Ministry_of_health_and_population_MOHP_Egypt.

13. Coakes SJ, Steed L. SPSS: Analysis Without Anguish Using SPSS Ion 14.0 For Windows. John Wiley \& Sons, Inc; 2009.

14. World Medical Association. Declaration of Helsinki. Ethical Principles for Medical Research Involving Human Subjects. World Medical Association; 2009.

15. Li X, Xu S, Yu M, et al. Risk factors for severity and mortality in adult COVID-19 inpatients in Wuhan. J Allergy Clin Immunol. 2020;146(1):110-118. doi:10.1016/j.jaci.2020.04.006

16. Bwire GM. Coronavirus: why men are more vulnerable to Covid-19 than women? SN Compr Clin Med. 2020;4:1-3.

17. Gao F, Zheng KI, Wang XB, et al. Obesity is a risk factor for greater COVID-19 severity. Diabetes Care. 2020;43(7):e72-e74. doi:10.2337/ dc20-0682

18. Wang X, Fang X, Cai Z, et al. Comorbid chronic diseases and acute organ injuries are strongly correlated with disease severity and mortality among COVID-19 patients: a systemic review and meta-analysis. Research. 2020;2020:2402961. doi:10.34133/2020/2402961

19. Zou X, Chen K, Zou J, Han P, Hao J, Han Z. Single-cell RNA-seq data analysis on the receptor ACE2 expression reveals the potential risk of different human organs vulnerable to $2019-\mathrm{nCoV}$ infection. Front Med. 2020;14(2):185-192. doi:10.1007/s11684-020-0754-0
20. Wei XS, Wang X, Niu YR, et al. Diarrhea is associated with prolonged symptoms and viral carriage in Corona virus disease 2019. Clin Gastroenterol Hepatol. 2020;18(8):1753-1759.e2. doi:10.1016/ j.cgh.2020.04.030

21. D’Amico F, Baumgart DC, Danese S, Peyrin-Biroulet L. Diarrhea during COVID-19 infection: pathogenesis, epidemiology, prevention, and management. Clin Gastroenterol Hepatol. 2020;18(8):16631672. doi:10.1016/j.cgh.2020.04.001

22. Anon. Diarrhoeal disease; 2020. Available from: https://www.who. int/news-room/fact-sheets/detail/diarrhoeal-disease. Accessed March $22,2020$.

23. Jin $\mathrm{X}$, Lian $\mathrm{J}, \mathrm{Hu} \mathrm{J}$, et al. Epidemiological, clinical and virological characteristics of 74 cases of coronavirus-infected disease 2019 (COVID-19) with gastrointestinal symptoms. Gut. 2020;69:10021009. doi:10.1136/gutjnl-2020-320926

24. Ramachandran P, Onukogu I, Ghanta S, et al. Gastrointestinal symptoms and outcomes in hospitalized Coronavirus disease 2019 patients. Dig Dis. 2020;38(5):373-379. doi:10.1159/000509774

25. O'Keefe JB. Predictors of disease duration and symptom course of outpatients with acute covid-19: a. Infection. 2020;21.

26. $\mathrm{Xu} \mathrm{XW,} \mathrm{Wu} \mathrm{XX,} \mathrm{Jiang} \mathrm{XG,} \mathrm{et} \mathrm{al.} \mathrm{Clinical} \mathrm{findings} \mathrm{in} \mathrm{a} \mathrm{group} \mathrm{of}$ patients infected with the 2019 novel coronavirus (SARS-Cov-2) outside of Wuhan, China: retrospective case series. BMJ. 2020;368: m606.

27. Wang $\mathrm{D}, \mathrm{Hu} \mathrm{B}, \mathrm{Hu} \mathrm{C}$, et al. Clinical characteristics of 138 hospitalized patients with 2019 novel coronavirus-infected pneumonia in Wuhan, China. [published online ahead of print, 2020 Feb 7]. JAMA. 2020;323:e201585. doi:10.1001/jama.2020.1585

28. Campioli CC, Cevallos EC, Assi M, Patel R, Binnicker MJ, O’Horo JC. Clinical predictors and timing of cessation of viral RNA shedding in patients with COVID-19. J Clin Virol. 2020;104577. doi:10.1016/j. jcv.2020.104577
Clinical and Experimental Gastroenterology

\section{Publish your work in this journal}

Clinical and Experimental Gastroenterology is an international, peerreviewed, open access, online journal publishing original research, reports, editorials, reviews and commentaries on all aspects of gastroenterology in the clinic and laboratory. This journal is indexed on American Chemical Society's Chemical Abstracts Service (CAS)

\section{Dovepress}

The manuscript management system is completely online and includes a very quick and fair peer-review system, which is all easy to use. Visit http://www.dovepress.com/testimonials.php to read real quotes from published authors. 